\title{
Crystal formation and size control of zeolitic imidazolate frameworks with mixed imidazolate linkers
}

\author{
Mikaela Gustafsson • Xiaodong Zou
}

Published online: 22 February 2012

(c) The Author(s) 2012. This article is published with open access at Springerlink.com

\begin{abstract}
The effect of synthesis parameters, such as batch composition and synthesis temperature, on the formation and crystal size of zeolitic imidazolate frameworks (ZIFs) using mixed linkers, imidazole (Im) and benzimidazole (bIm), were studied by X-ray powder diffraction (XRPD) and scanning electron microscopy (SEM). ZIF-7 and ZIF-62 were identified as the main phases and a phase diagram relating the batch composition and the final product was constructed based on XRPD and SEM. The range of batch composition giving rise to ZIF-7 and ZIF-62 was identified from the phase diagram. Changes of crystal size and morphology of ZIF-7 and ZIF-62 in terms of batch composition and synthesis temperature were studied by SEM and different trends were observed for the two ZIFs. Thermogravimetric analysis (TGA) and in situ XRPD showed that ZIF-62 possesses a similar high thermal stability as ZIF-7.
\end{abstract}

Keywords Zeolitic imidazolate framework . Metal-organic framework · Crystal engineering · Phase diagram - Crystal size and morphology control

Electronic supplementary material The online version of this article (doi:10.1007/s10934-012-9574-1) contains supplementary material, which is available to authorized users.

\footnotetext{
M. Gustafsson $(\varangle) \cdot X$. Zou $(\bowtie)$

Berzelii Centre EXSELENT on Porous Materials and Inorganic and Structural Chemistry, Department of Materials and Environmental Chemistry, Stockholm University, 10691 Stockholm, Sweden

e-mail: mikaela.gustafsson@mmk.su.se

X. Zou

e-mail: xzou@mmk.su.se
}

\section{Introduction}

Zeolitic imidazolate frameworks (ZIFs) are a sub-class of metal-organic frameworks (MOFs) that have higher surface areas than zeolites and higher thermal, hydrothermal and chemical stability than most of MOFs. They are one of the most interesting and promising classes of porous materials that show great potential in gas storage, separation and catalysis [1]. ZIFs are built up by metal ions $\left(\mathrm{M}=\mathrm{Zn}^{2+}\right.$ or $\left.\mathrm{Co}^{2+}\right)$ that are linked to four imidazolate (Im). Each Im is coordinated to two metal ions through their nitrogen atoms. Since the M-Im-M angle is $145^{\circ}$, similar to the $\mathrm{Si}-\mathrm{O}-\mathrm{Si}$ angle in zeolites [2], extended 3D frameworks that possess identical tetrahedral topologies of zeolites are expected for ZIFs. The combination of fourcoordinated transition metal ions and imidazolate-based linkers opened up new opportunities to synthesize porous materials with large pores, high surface areas and tunable functionalities [1]. It overcomes the limitation of zeolite chemistry to only a few framework-forming elements such as silicon, aluminum and oxygen. In addition, different imidazolate linkers may be combined to obtain new ZIFs with hetero-linkers. About $20 \%$ of all known ZIFs contain hetero-linkers [3, 4], for example ZIF-62 with imidazole and benzimidazole [3], ZIF-69 with 5-chloroimidazole and nitroimidazole [3, 4], and ZIF-70 with imidazole and nitroimidazole [3, 4].

Due to the robust imidazolate linkers and the strong bonding between the transition metal ions and imidazolates, ZIFs often show high stability towards heat, moisture and solvents and are promising candidates for industrial applications [2, 3]. For example, the structure of ZIF-8 was retained in boiling benzene, methanol and water for several days [2]. The great capacities of ZIFs to adsorb, store and separate gases [4] as well as hydrocarbons [5] have made 
them promising candidates as molecular sieves [6-8], films [9] and membranes [10-13]. ZIFs have for example high affinity for $\mathrm{CO}_{2}$ [14]. ZIF-69 can store $\mathrm{CO}_{2}$ to a greater extent than the industrially used absorbent BPL carbon [3]. ZIF-7 [2] can separate $\mathrm{CO}_{2}$ from $\mathrm{N}_{2}$ in combustion gas streams at relative low pressures and moderate temperatures [15], and alkanes and alkenes through gate-opening process [16, 17]. ZIF-7 has also been synthesized as films on alumina supports and used as membranes in sensors and for gas separation [9], for example separating $\mathrm{H}_{2}$ from $\mathrm{CO}_{2}$ $[18,19]$.

The properties of porous materials depend not only on the framework structure, pore size and surface areas, but also on the crystal size and morphology. Size and morphology control is thus important, especially for applications in gas separation and catalysis. It is important to identify and optimize the synthesis parameters that control the crystal formation, size and morphology. Recently Venna et al. [20] studied the structural evolution of ZIF-8 as a function of synthesis time. Cravillon et al. [21] reported the crystal growth and control of nano- and microcrystal formation of ZIF-8. Even though many ZIFs with interesting properties have been synthesized, to the best of our knowledge, no systematic study has been reported yet on the crystal formation, size and morphology control by changing the synthesis parameters such as batch composition and temperature.

Here, we present a study of how different synthesis parameters, such as batch composition and temperature, influence on the crystal formation, size and morphology of zeolitic imidazolate frameworks (ZIFs) when mixed linkers, imidazole ( $\mathrm{Im}$ ) and benzimidazole (bIm), were used. A phase diagram, based on XRPD and SEM, was constructed in order to relate the batch composition with the formation of different ZIF phases. The crystal size and morphology of the two main products, ZIF-7 and ZIF-62, were further studied by scanning electron microscopy (SEM).

\section{Experimental}

\subsection{Synthesis of ZIFs}

The ZIFs were prepared using solvothermal synthesis. We used $\mathrm{Zn}\left(\mathrm{NO}_{3}\right)_{2} \cdot 6 \mathrm{H}_{2} \mathrm{O}$ as the metal source, imidazole ( $\left.\mathrm{ImH}\right)$ and benzimidazole (bImH) as the linkers, and $N, N$ dimethylformamide (DMF) as the solvent. In order to study the influence of the metal to linker ratio and the ratio of the hetero-linkers on the formation of ZIFs, we designed the synthesis batch compositions as given in Table 1. Three different metal $(\mathrm{Zn})$ to linker $(\mathrm{L}=\mathrm{Im}+\mathrm{bIm})$ ratios, $\mathrm{Zn}: \mathrm{L}=1: 15,1: 10$ and 1:5, were applied. For each $\mathrm{Zn}: \mathrm{L}$ ratio, thirteen different Im to bIm ratios were tested. First,
Table 1 Batch compositions used for the syntheses of ZIFs

\begin{tabular}{llll}
\hline Im:bIm & Zn:L & & \\
\cline { 2 - 4 } & $1: 15$ & $1: 10$ & $1: 5$ \\
\hline $1: 0$ & {$[1: 15: 0]$} & {$[1: 10: 0]$} & {$[1: 5: 0]$} \\
$9: 1$ & {$[1: 13.5: 1.5]$} & {$[1: 9: 1]$} & {$[1: 4.5: 0.5]$} \\
$7: 1$ & {$[1: 13.13: 1.88]$} & {$[1: 8.75: 1.25]$} & {$[1: 4.38: 0.63]$} \\
$5: 1$ & {$[1: 12.5: 2.5]$} & {$[1: 8.33: 1.67]$} & {$[1: 4.17: 0.83]$} \\
$3: 1$ & {$[1: 11.25: 3.75]$} & {$[1: 7.5: 2.5]$} & {$[1: 3.75: 1.25]$} \\
$3: 2$ & {$[1: 9: 6]$} & {$[1: 6: 4]$} & {$[1: 3: 2]$} \\
$1: 1$ & {$[1: 7.5: 7.5]$} & {$[1: 5: 5]$} & {$[1: 2.5: 2.5]$} \\
$2: 3$ & {$[1: 6: 9]$} & {$[1: 4: 6]$} & {$[1: 2: 3]$} \\
$1: 3$ & {$[1: 3.75: 11.25]$} & {$[1: 2.5: 7.5]$} & {$[1: 1.25: 3.75]$} \\
$1: 4$ & {$[1: 3: 12]$} & {$[1: 2: 8]$} & {$[1: 1: 4]$} \\
$1: 7$ & {$[1: 1.88: 13.13]$} & {$[1: 1.25: 8.75]$} & {$[1: 0.63: 4.38]$} \\
$1: 9$ & {$[1: 1.5: 13.5]$} & {$[1: 1: 9]$} & {$[1: 0.5: 4.5]$} \\
$0: 1$ & {$[1: 0: 15]$} & {$[1: 0: 10]$} & {$[1: 0: 5]$} \\
\hline
\end{tabular}

Each batch is denoted with the corresponding molar ratio $\mathrm{Zn}: \mathrm{Im}: \mathrm{bIm}$ as [Zn:Im:bIm]. The numbers in brackets correspond to the batch compositions of performed syntheses

we prepared three stock solutions by dissolving the reagents in DMF: $1 \mathrm{M}$ of ImH, $1 \mathrm{M}$ of bImH and $15 \mathrm{mM}$ of $\mathrm{Zn}\left(\mathrm{NO}_{3}\right)_{2} \cdot 6 \mathrm{H}_{2} \mathrm{O}$. Then appropriate volumes of the stock solutions, calculated according to the zinc:ligand $(\mathrm{Zn}: \mathrm{L})$ and imidazole:benzimidazole (Im:bIm) ratios in Table 1, were mixed together in a Teflon-lined autoclave to get a total volume of $4 \mathrm{~mL}$ ( $50 \mathrm{mmol}$ of DMF). An example of the synthesis is as follows: $0.01 \mathrm{~mL}$ stock solution of $\mathrm{Zn}\left(\mathrm{NO}_{3}\right)_{2} \cdot 6 \mathrm{H}_{2} \mathrm{O}(0.044 \mathrm{~g}, 0.150 \mathrm{mmol}), 0.375 \mathrm{~mL}$ of the Im stock solution $(0.003 \mathrm{~g}, 0.375 \mathrm{mmol}), 0.375 \mathrm{~mL}$ of the bIm stock solution $(0.005 \mathrm{~g}, 0.375 \mathrm{mmol})$ were mixed in a Teflon-lined autoclave and $3.24 \mathrm{~mL}$ of DMF was added to reach a total volume of $4 \mathrm{~mL}$ ( $50 \mathrm{mmol})$. The final batch composition $\mathrm{Zn}: \mathrm{Im}: \mathrm{bIm}: \mathrm{DMF}$ was $1: 2.5: 2.5: 333$, which is denoted as [1:2.5:2.5] in Table 1. The reaction solution was stirred for $1 \mathrm{~h}$ and then placed in a preheated oven at $110{ }^{\circ} \mathrm{C}$ for 4 days. The solid was washed with DMF and dried at room temperature. In order to check the reproducibility, we repeated most of the syntheses with the different batch compositions at $110{ }^{\circ} \mathrm{C}$ for more than one time (1-3 times). Some synthesis series were also performed at $85{ }^{\circ} \mathrm{C}$ to study the temperature effect on the formation and size of ZIF-7 and ZIF-62.

\subsection{Characterization}

\subsubsection{X-ray powder diffraction (XRPD) analysis}

XRPD was performed on a PANalytical X'Pert PRO diffractometer equipped with a Pixel detector and using $\mathrm{Cu}$ $\mathrm{K} \alpha_{1}$ radiation $(\lambda=1.5406 \AA)$. All products obtained from 
the synthesis series with various batch compositions and temperatures were analyzed by XRPD. Each sample was ground prior to data collection and dispersed uniformly on zero-background Si plates. The XRPD pattern was compared with those of known ZIFs.

\subsubsection{Single crystal $X$-ray diffraction}

Single crystal X-ray diffraction data of a ZIF-62 crystal from the sample synthesized with the batch composition [1:13.5:1.5] at $110{ }^{\circ} \mathrm{C}$ were collected at $298 \mathrm{~K}$ on an XCalibur diffractometer equipped with a CCD camera using Mo $_{\alpha}(\lambda=0.7107 \AA)$ radiation. The structure was solved and refined using the program SHELX [22].

\subsubsection{Scanning electron microscopy (SEM)}

The size and morphology of the ZIF samples were investigated on a JEOL JSM-7000F field emission SEM operated at $3 \mathrm{kV}$. The samples were pre-coated with a thin gold film.

\subsubsection{Thermogravimetric analysis (TGA)}

TGA was performed on a sample synthesized with the batch composition [1:13.5:1.5] at $110^{\circ} \mathrm{C}$ under a nitrogen atmosphere from 30 to $650{ }^{\circ} \mathrm{C}$ with a heating rate of $1{ }^{\circ} \mathrm{C} /$ min using a high-resolution thermogravimetric analyzer (PERKIN ELMER TGA 7).

\subsubsection{In situ X-ray powder diffraction (XRPD)}

In situ XRPD of ZIF-62 was performed on the sample synthesized with the batch composition [1:13.5:1.5] at $110{ }^{\circ} \mathrm{C}$ from $\mathrm{RT}$ to $450{ }^{\circ} \mathrm{C}$ in a nitrogen atmosphere ( $\sim 2$ bar) on a PANalytical X'Pert PRO MPD diffractometer equipped with an Anton-Parr XRK900 reaction chamber, using $\mathrm{Cu} \mathrm{K}_{\alpha}$ radiation $(\lambda=1.5418 \AA)$ and variable slits. The heating rate was $2{ }^{\circ} \mathrm{C} / \mathrm{min}$ and the temperature was equilibrated for $2 \mathrm{~min}$ prior to each data collection. An open Macor glass ceramic sample holder with a sieve-like bottom (pore size $0.5 \mathrm{~mm}$ ) was used. The temperature was controlled by a thermo-couple ca. $3 \mathrm{~mm}$ from the sample.

\section{Results and discussion}

3.1 Effect of the batch compositions on the final products

XRPD showed that ZIF-7 [ $\left.\mathrm{Zn}(\mathrm{bIm})_{2} \cdot 3 \mathrm{H}_{2} \mathrm{O}\right]$ and ZIF-62 $\left[\mathrm{Zn}(\mathrm{Im})_{1.75}(\mathrm{bIm})_{0.25} \cdot 0.5 \mathrm{DMF}\right]$ were the two main phases in the products. ZIF-7 is trigonal with the space group $R-3$ and hexagonal unit cell $a=22.989(3) \AA$ and $c=15.763(3) \AA$ [2]. ZIF-62 is orthorhombic with the space group Pbca and unit cell $a=15.6620(14) \AA, b=15.6620(14) \AA$ and $c=18.2073(19) \AA$. ZIF-7 adopts the sod topology and contains one unique $\mathrm{Zn}$ atom, which is coordinated to four bIms (Fig. 1a) [2]. ZIF-62 adopts the cag topology and contains two unique $\mathrm{Zn}$ atoms, each is coordinated to three Ims and one linker disordered between Im (60\%) and bIm (40\%) (Fig. 1b) [3].

Two typical XRPD patterns of the ZIF-7 and ZIF-62 samples are shown in Fig. 2. The XRPD patterns of the samples synthesized at $110{ }^{\circ} \mathrm{C}$ from the synthesis series with $\mathrm{Zn}: \mathrm{L}$ ratios of 1:15, 1:10 and 1:5 are shown in Fig. 3. Most of the XRPD patterns resemble that of ZIF-7 or ZIF62. Samples synthesized with only imidazole were identified as ZIF-4 $\left(\mathrm{Zn}(\operatorname{Im})_{2}\right)$ [2]. Since ZIF-62 has the same space group and similar unit cell parameters as ZIF-4, we also used single crystal X-ray diffraction to confirm the phase. Our single crystal X-ray diffraction data gave the orthorhombic space group Pbca (61) with $a=15.5848(6) \AA$,

Fig. 1 The structure illustrations of ZIF-7, $\left[\mathrm{Zn}(\mathrm{bIm})_{2} \cdot 3 \mathrm{H}_{2} \mathrm{O}\right][2]$ and ZIF-62, $\left[\mathrm{Zn}(\mathrm{Im})_{1.75}\right.$ $\left.(\mathrm{bIm})_{0.25} \cdot 0.5 \mathrm{DMF}\right][3]$

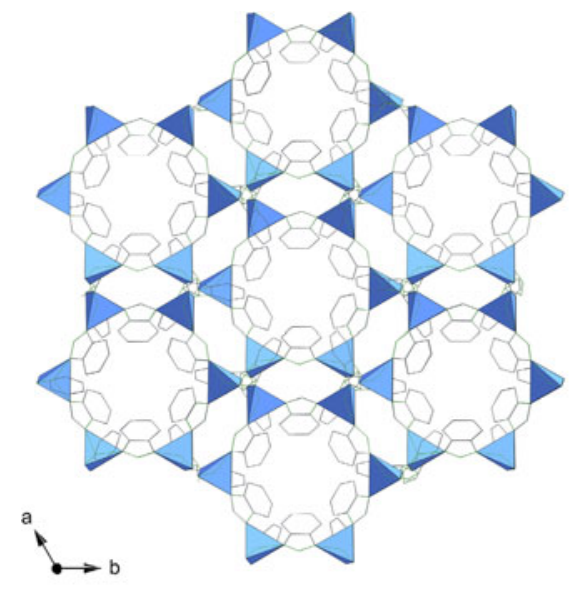

ZIF-7 (sod)

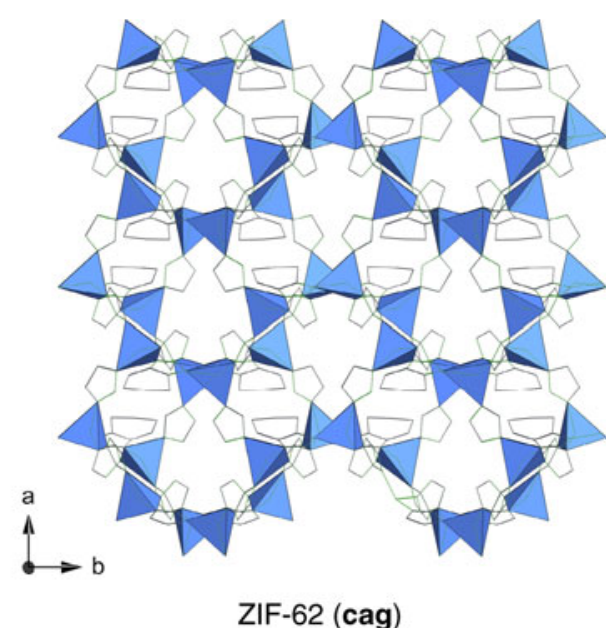

ZIF-62 (cag) 

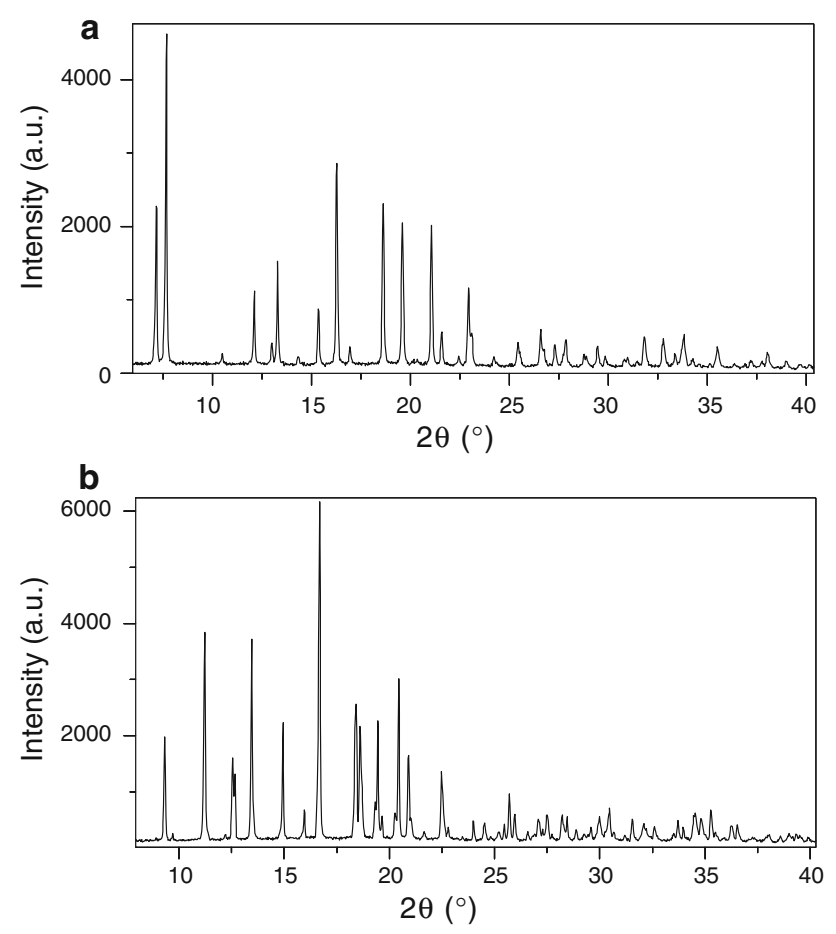

Fig. 2 XRPD patterns of a ZIF-7 obtained at Zn:L of 1:5 and Im:bIm of $1: 7$, or [Zn:Im:bIm] of $[1: 0.63: 4.38]$ at $110^{\circ} \mathrm{C}$ and b ZIF-62 obtained at $\mathrm{Zn}: \mathrm{L}$ of $1: 5$ and $\mathrm{Im}: \mathrm{bIm}$ of $5: 1$, or [Zn:Im:bIm] of [1:4.17:0.83] at $110{ }^{\circ} \mathrm{C}$. The background was subtracted from each XRPD pattern

$b=15.7370(5) \AA, c=18.2808(7) \AA$. The structure was identical to that of ZIF-62 [3].

As shown in Fig. 3 and Table S1, ZIF-62 could be synthesized at $110{ }^{\circ} \mathrm{C}$ within a wide range of zinc to linker ratios, from $\mathrm{Zn}: \mathrm{L}$ of $1: 15$ to $1: 5$, as long as the $\mathrm{Im}:$ bIm ratio is no less than 3:1. With higher $\mathrm{Zn}$ concentration (Zn:L > 1:5), including the ideal composition [1:1.75:0.25] of ZIF-62, no ZIF-62 was obtained. For Zn:L of 1:15 and $1: 10$, there is a clear boarder between the batch compositions that form ZIF-7 and ZIF-62 (Fig. 3a, b), while for $\mathrm{Zn:L}$ of 1:5, both ZIF-7 and ZIF-62 were formed within the same batch, i.e. [1:3:2] (Fig. 3c). No solid was observed at the batch compositions of [1:9:6] for $\mathrm{Zn}: \mathrm{L}$ of 1:15.

The formation of the ZIFs can be better understood by constructing a ternary phase diagram, from which the composition range that forms a target compound can be identified. A phase diagram, shown in Fig. 4, was constructed based on the XRPD data of the samples synthesized at $110^{\circ} \mathrm{C}$ at the $\mathrm{Zn}: \mathrm{L}$ ratios of $1: 15,1: 10$ and 1:5, respectively (Fig. 3). The phase diagram gives a clear overview about the formation of different phases at various batch compositions. The batch composition regions that form ZIF-7 and ZIF-62 are well separated. ZIF-7 was formed within a wider Im:bIm range compared to ZIF-62, as illustrated clearly in Fig. 4. ZIF-4 was obtained when imidazole was used as the only linker. When benzimidazole was introduced in the synthesis at $\mathrm{Zn}: \mathrm{L}$ of $1: 15$, ZIF-62 was formed up to $23 \%$ of benzimidazole. At the benzimidazole concentration of $37 \%$, no solid was obtained. ZIF-7 was formed with the benzimidazole concentration between 47 and $100 \%$. At the $\mathrm{Zn}: \mathrm{L}$ of $1: 10$, ZIF-62 was formed up to $23 \%$ of benzimidazole. ZIF-7 could be obtained with the concentration of benzimidazole between 36 and $100 \%$. At the $\mathrm{Zn}: \mathrm{L}$ of 1:5, ZIF-62 was formed up to $21 \%$ of benzimidazole. At the benzimidazole concentration of $33 \%$, ZIF-7 was formed together with a minor amount of ZIF-62. With the benzimidazole concentration above $33 \%$, only ZIF-7 was formed.

It is interesting to note that only one (ZIF-4) of the known ZIFs with the composition $\mathrm{Zn}(\mathrm{Im})_{2}$ (ZIF-1, -2, -3, $-4,-6,-10$ and -64$)[2,3]$ was obtained from our synthesis series. This may be due to the different zinc sources $\left(\mathrm{Zn}\left(\mathrm{NO}_{3}\right)_{2} \cdot 6 \mathrm{H}_{2} \mathrm{O}\right.$ with respective to $\left.\mathrm{Zn}\left(\mathrm{NO}_{3}\right)_{2} \cdot 4 \mathrm{H}_{2} \mathrm{O}\right)$, different $\mathrm{Zn}: \mathrm{Im}$ ratios (low with respective to high $\mathrm{Zn}$ concentrations) and different heating time (4 with respective to 1 day for ZIF-1) for our synthesis and those published by Yaghi's group. We did neither obtain ZIF-11 (Zn(bIm) $)_{2}$, which was synthesized in $N, N$-diethylformamide (DEF) at $100{ }^{\circ} \mathrm{C}$ for 4 days with $\mathrm{Zn}$ :bIm ratio of 1:18 [2]. The facts that ZIF-7 and ZIF-62 could be formed in a wide range of batch compositions which are far away from their ideal molar ratios (marked by circles in Fig. 4), indicate that they were thermodynamically most stable in the process of nucleation.

\subsection{Effects of synthesis parameters on crystal size and morphology}

X-ray powder diffraction only tells the presence of different crystalline phases in a sample, but does not show possible variations of size and morphology of the crystals. The size and morphology of the ZIF-7 and ZIF-62 samples were therefore studied closely by scanning electron microscopy, in order to find out how variations of batch composition and temperature affect the size and shape of the crystals.

SEM images of the studied ZIF-7 and ZIF-62 samples are given in Tables S2-S3. Most of the ZIF-7 crystals have a rhombic dodecahedral shape. The average size of ZIF-7 ranges from 0.5 to $10 \mu \mathrm{m}$. We could identify that the size of the ZIF-7 crystals synthesized at $110{ }^{\circ} \mathrm{C}$ was mainly affected by the ratio between bIm and $\mathrm{Zn}$ as shown in Fig. 5a-g. The crystal size changed with the bIm:Zn ratio in a non-linear manner, as illustrated in the graph in Fig. 5. At a low ratio of $\mathrm{bIm} / \mathrm{Zn}(=2.25)$, the crystal size of ZIF-7 was less than $1 \mu \mathrm{m}$. The crystal size increased first slightly with higher $\mathrm{bIm} / \mathrm{Zn}$ ratio, and then drastically from 1.2 to $10 \mu \mathrm{m}$ as the $\mathrm{bIm} / \mathrm{Zn}$ ratio increased from 3.75 to 4.5 
Fig. 3 XRPD patterns of the samples synthesized at $110{ }^{\circ} \mathrm{C}$ from synthesis series with $\mathrm{Zn}: \mathrm{L}$ of $\mathbf{a}$ 1:15, b 1:10 and $\mathbf{c}$ 1:5. The corresponding batch composition and identified crystalline phase(s) are given next to each XRPD pattern. When two phases are listed for the same sample, the first phase is the major one. The amount of sample obtained at the batch composition [1:11.25:3.75] was too small for XRPD and no solid phase was obtained for the batch composition [1:9:6]. Therefore XRPD patterns corresponding to these two compositions are missing here. The background was subtracted for clarity

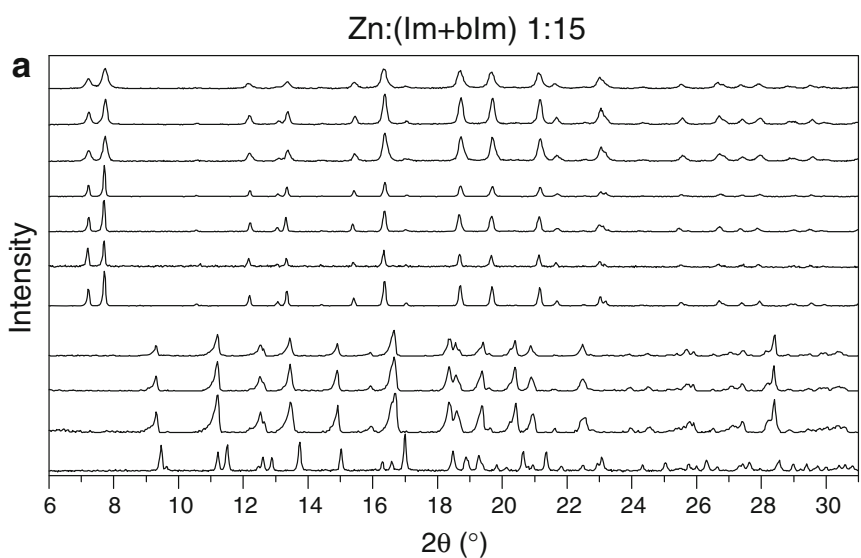

Zn:Im:blm Phase

1:0:15 ZIF-7

1:1.5:13.5 ZIF-7

1:1.88:13.13 ZIF-7

1:3:12 ZIF-7

1:3.75:11.25 ZIF-7

1:6:9 ZIF-7

1:7.5:7.5 ZIF-7

1:12.5:2.5 ZIF-62

1:13.13:1.88 ZIF-62

1:13.5:1.5 ZIF-62

1:15:0 ZIF-4

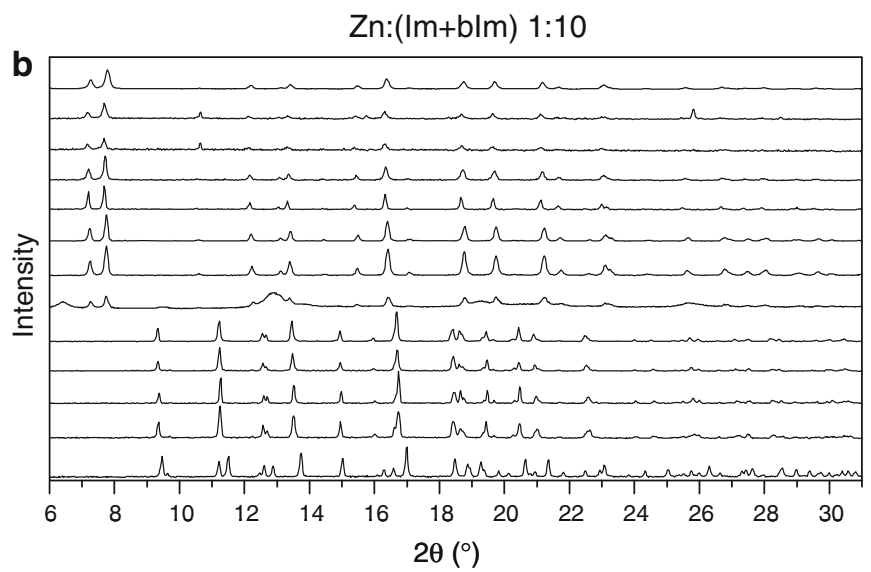

Zn:Im:blm Phase

1:0:10 ZIF-7

1:1:9 ZIF-7

1:1.25:8.75 ZIF-7

1:2:8 ZIF-7

1:2.5:7.5 ZIF-7

1:4:6 ZIF-7

1:5:5 ZIF-7

1:6:4 (ZIF-7)

1:7.5:2.5 ZIF-62

1:8.33:1.67 ZIF-62

1:8.75:1.25 ZIF-62

1:9:1 ZIF-62

1:10:0 ZIF-4

Zn:(Im+blm) 1:5

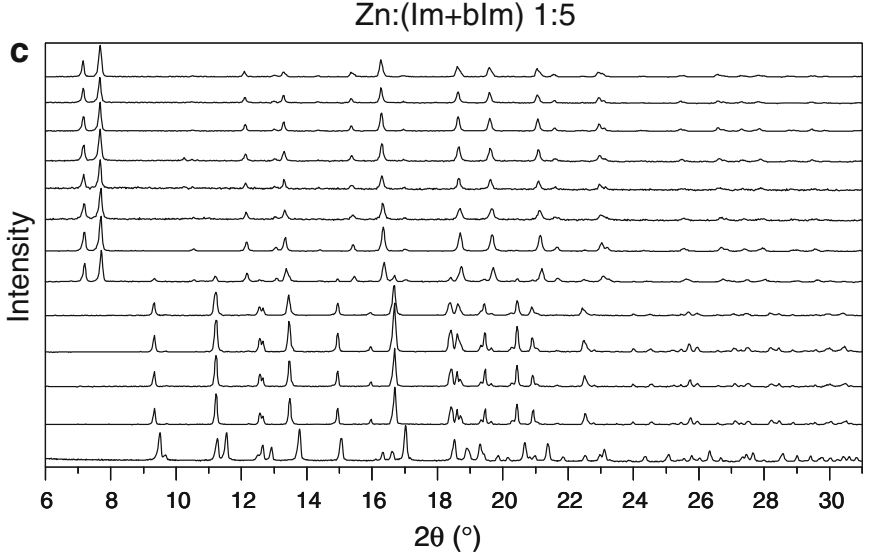

Zn:Im:blm Phase

1:0:5 ZIF-7

1:4.5:0.5 ZIF-7

1:0.63:4.38 ZIF-7

1:1:4 ZIF-7

1:1.25:3.75 ZIF-7

1:2:3 ZIF-7

1:2.5:2.5 ZIF-7

1:3:2 ZIF-7+ZIF-62

1:3.75:1.25 ZIF-62

1:4.17:0.83 ZIF-62

1:4.38:0.63 ZIF-62

1:4.5:0.5 ZIF-62

1:5:0 ZIF-4
(Fig. 5b-c). Further increase of the bIm/Zn ratio did not increase the crystal size. Instead, the crystal size decreased continuously from $10 \mu \mathrm{m}$ down to $0.8 \mu \mathrm{m}$ at the $\mathrm{bIm} / \mathrm{Zn}$ ratio of 12 (Fig. 5c-g). The batch composition of [1:0.5:4.5] gave the largest crystals (Fig. 5c). As the bIm/ $\mathrm{Zn}$ ratio increased from 6 to 12 , the crystals became more aggregated (Fig. 5d-g).

The crystal size of ZIF-7 also changed with the synthesis temperature, as shown in Fig. 6. When the temperature decreased from 110 to $85^{\circ} \mathrm{C}$, the changes in crystal size are rather small. The average crystal size increased slightly from 2.4 to $4.7 \mu \mathrm{m}$ for the samples with the batch composition [1:6:9] and from 0.8 to $1.3 \mu \mathrm{m}$ for the samples with the batch composition [1:3:12], and showed no significant change for samples with the batch composition [1:3.75:11.25]. This indicates that the synthesis temperature has a minor influence on the crystal size of ZIF-7. In general, the crystals became better-shaped, with sharper edges and smoother surfaces, and less aggregated as the crystal size increased. No obvious change of the crystal shape was observed when the synthesis temperature was decreased from 110 to $85^{\circ} \mathrm{C}$. We also found that the 


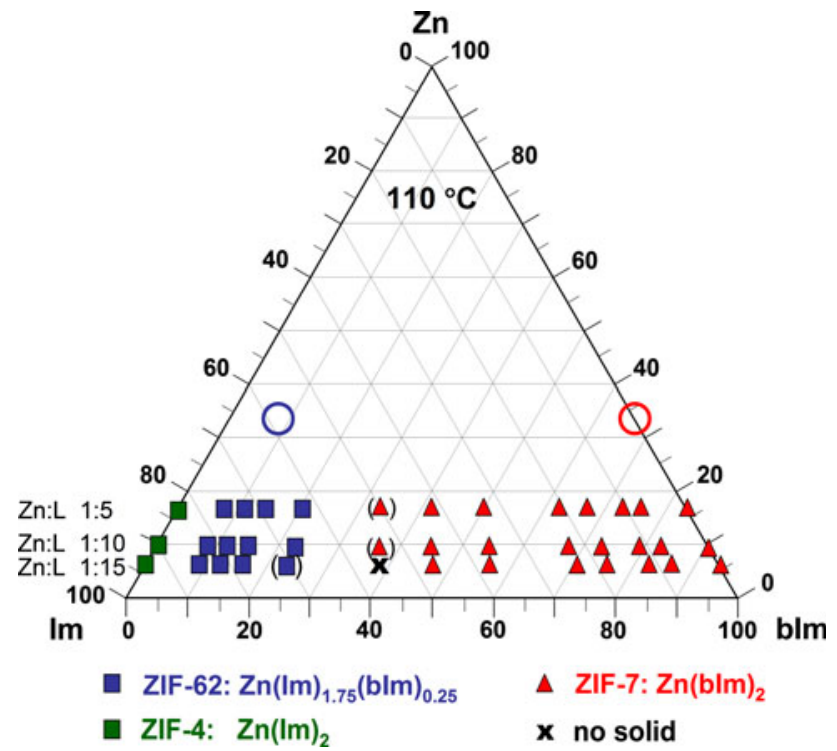

Fig. 4 The phase diagram constructed from the phases identified from the XRPD patterns in Fig. 3. ZIF-62 is marked by blue square, ZIF-7 in red triangle, ZIF-4 in green square and where no solid was formed are marked by a cross. The blue square marked by brackets indicates too small yields for XRPD. Red triangles marked by brackets indicate the presence of other phases (Color figure online) variation of $\mathrm{Zn}$ concentrations did not affect the size or shape of the crystals significantly, as shown in Table S2.

The crystals of ZIF-62 were significant larger $(10-500 \mu \mathrm{m})$ than those of ZIF-7 $(0.5-10 \mu \mathrm{m})$. Both isolated crystals and crystal aggregates were observed (Table S3). Most of the large ZIF-62 crystals have a pseudo-octahedral shape, with two opposite corners of the octahedron being truncated (Fig. 7a-c, f). The size of ZIF-62 was the largest $(500 \mu \mathrm{m})$ at the batch composition of [1:11.25:3.75] and synthesis temperature of $110^{\circ} \mathrm{C}$ (Fig. 7c). The average crystal size decreased significantly from 500 to $100 \mu \mathrm{m}$ with the increase of the Im:bIm ratio from [1:11.5:1.5] to [1:13.5:1.5] (Fig. 7a-c). With higher $\mathrm{Zn}$ concentrations at the $\mathrm{Zn}: \mathrm{L}$ ratio of $1: 10$ and $1: 5$, the crystals of $\mathrm{ZIF}-62$ were much smaller $(10-160 \mu \mathrm{m})$ (Table S3). Within the same $\mathrm{Zn}: \mathrm{L}$ ratio, the average crystal size increased with the decrease of the $\mathrm{Im} / \mathrm{Zn}$ ratio and increase of the $\mathrm{bIm} / \mathrm{Zn}$ ratio. For all the three $\mathrm{Zn}: \mathrm{L}$ ratios, the crystals of ZIF-62 were the largest when the Im:bIm ratio in the synthesis batch was 3:1.

The crystals of ZIF-62 synthesized at $110^{\circ} \mathrm{C}$ (Fig. 7a-c) were much larger compared with those formed at $85^{\circ} \mathrm{C}$ (Fig. 7d-f). The average crystal size also increased with the decrease of the Im:bIm ratio, but much more slowly
Fig. 5 A graph and SEM images showing how the crystal sizes of ZIF-7 changed with the $\mathrm{bIm} / \mathrm{Zn}$ molar ratio at $110^{\circ} \mathrm{C}$ (the size range of crystals within each batch is marked). Each point in the graph is correlated to an image, denoted $(a)-(g)$. The crystal size increased between $(a)-(c)$ and decreased from the maximum $10 \mu \mathrm{m}$ in (c) down to $0.8 \mu \mathrm{m}$ in $(g)$. As the crystals became larger they had also more well-defined shapes with sharper edges. The crystals of ZIF-7 became more aggregated and less well-shaped as the $\mathrm{bIm} / \mathrm{Zn}$ ratio increased from $6(d)$ to $12(g)$
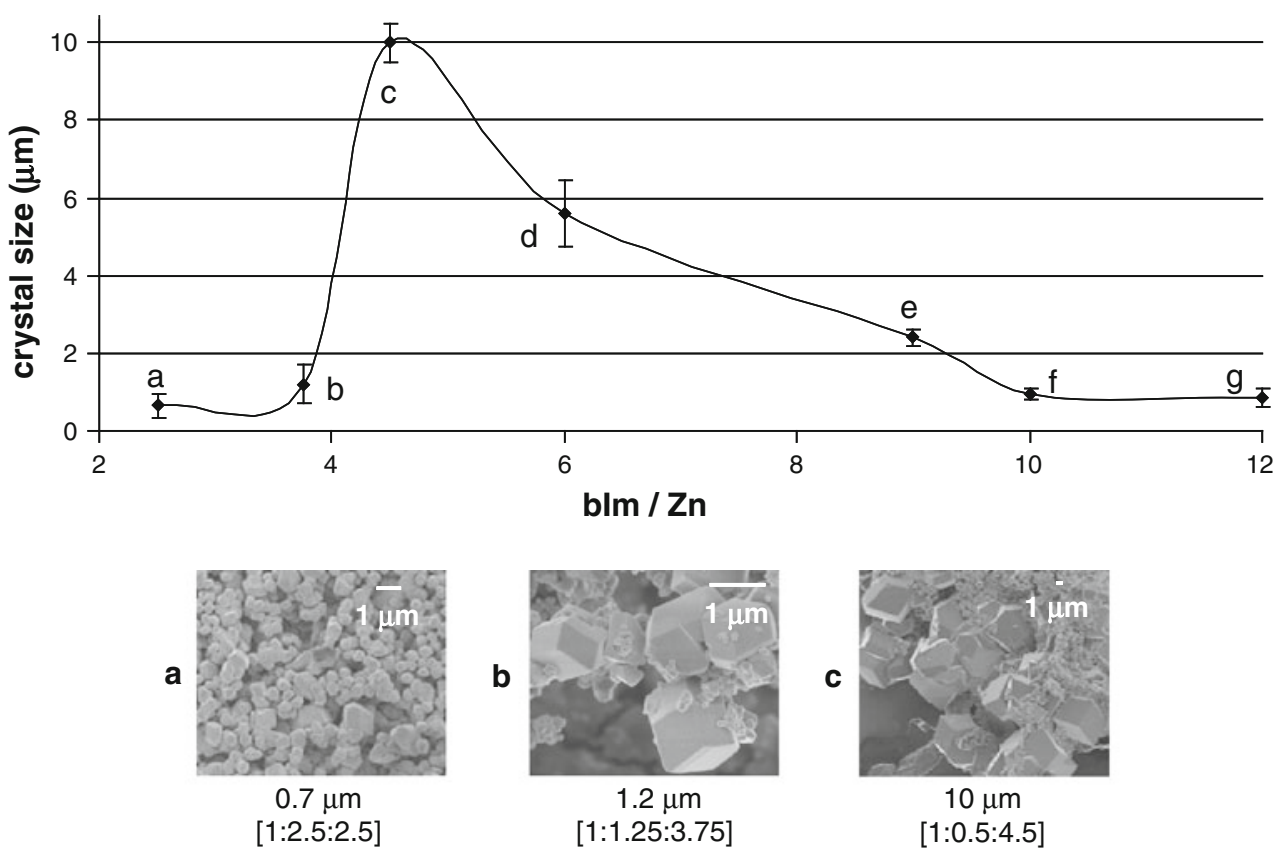

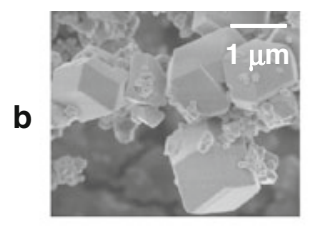

$1.2 \mu \mathrm{m}$

[1:1.25:3.75]

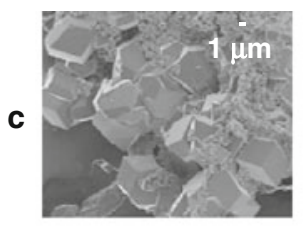

$10 \mu \mathrm{m}$

[1:0.5:4.5]

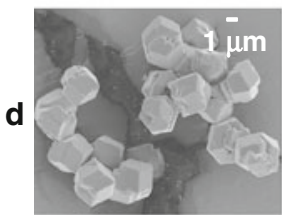

$5.6 \mu \mathrm{m}$ [1:4:6]

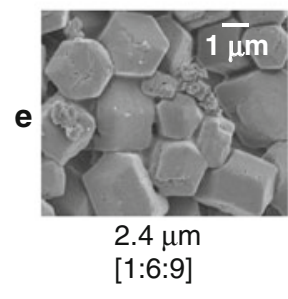

$2.4 \mu \mathrm{m}$
$[1: 6: 9]$

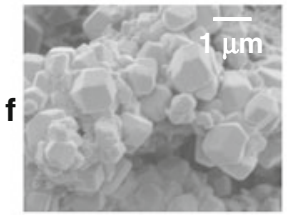

$1.0 \mu \mathrm{m}$

[1:0:10]

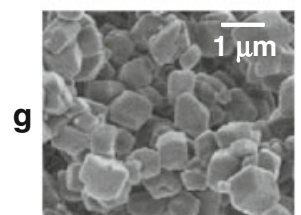

$0.8 \mu \mathrm{m}$

[1:3:12] 
Fig. 6 SEM images of ZIF-7 crystals synthesized using the $\mathrm{Zn}: \mathrm{L}$ ratio of $1: 15$ at (a-c) $110{ }^{\circ} \mathrm{C}$ and $(\mathbf{d}-\mathbf{f}) 85^{\circ} \mathrm{C}$ with Im:bIm ratio of (a, d) 2:3, $(\mathbf{b}, \mathbf{e}) 1: 3$ and $(\mathbf{c}, \mathbf{f}) 1: 4$
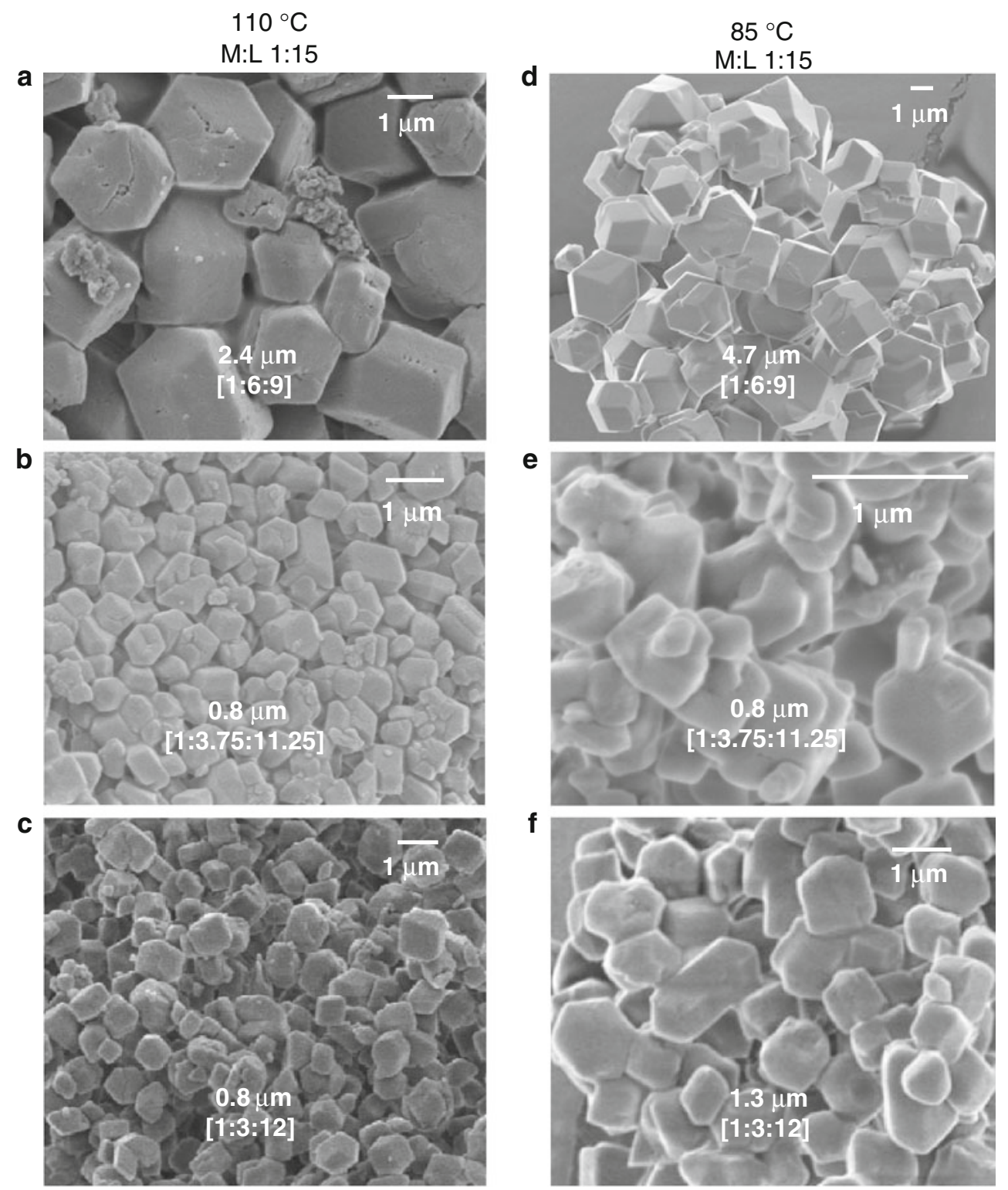

than at $110{ }^{\circ} \mathrm{C}$, only from 12 to $25 \mu \mathrm{m}$. In addition, the morphology of the crystals changed with the decrease of the Im: bIm ratio, from crystal aggregates to isolated single crystals (Fig. 7d-f). No obvious changes of the crystal shape were observed when the synthesis temperature was decreased from 110 to $85^{\circ} \mathrm{C}$.

\subsection{Thermal stability of ZIF-62}

The thermal stability of ZIF-7 has been reported by Yaghi's group by thermogravimetric analysis (TGA) [2]. The TGA curve of ZIF-7 gave the first weight-loss of $15.8 \%$ between 130 and $275^{\circ} \mathrm{C}$ corresponding to the loss of the water molecules. The second weight-loss started after $460{ }^{\circ} \mathrm{C}$ corresponding to the decomposition of the framework. We performed the TGA analysis on ZIF-62 in order to compare its thermal stability with that of ZIF-7. The TGA curve of ZIF-62 showed the first weight-lost of $15 \%$ between 180 and $320{ }^{\circ} \mathrm{C}$, corresponding to the loss of the guest DMF molecules in the pores $(0.5 \mathrm{DMF}$ per formula, calculated 15\%) (Fig. 8). These temperatures were higher than those for ZIF-7, due to the larger size of DMF compared to that of water. The second weight-loss started at $480{ }^{\circ} \mathrm{C}$ corresponding to the decomposition of the framework. The slightly higher thermal stability of ZIF-62 than that of ZIF-7 may be due to the denser framework of ZIF-62. In order to verify up to which temperature the framework structure of ZIF-62 was retained, an in situ 

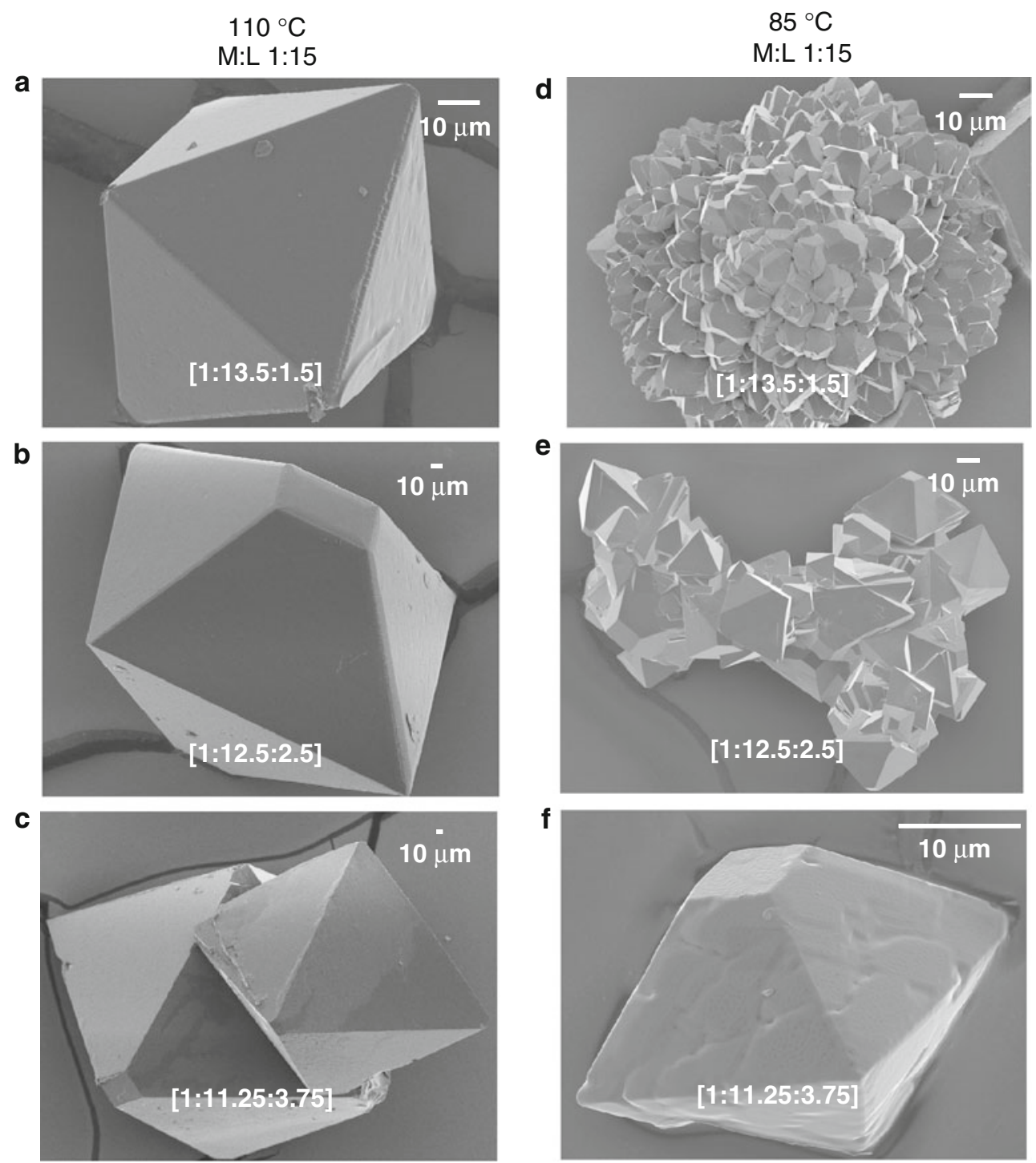

Fig. 7 SEM images showing the crystal size and morphology of ZIF-62 at (a-c) $110{ }^{\circ} \mathrm{C}$ and (d-f) $85{ }^{\circ} \mathrm{C}$. SEM images from three batch compositions are given, with (a, d) [1:13.5:1.5], (b, e) [1:12.5:2.5] and (c, f) [1:11.25:3.75]

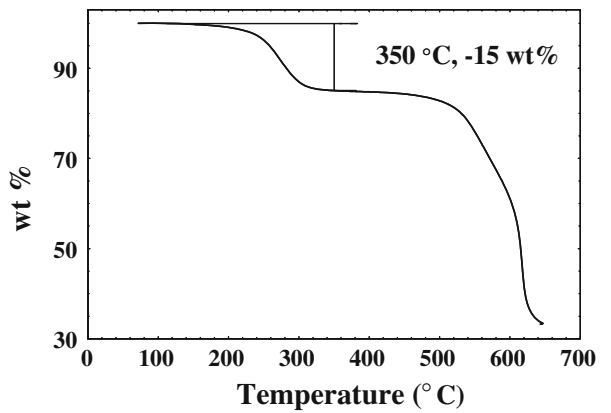

Fig. 8 TG curve of ZIF-62, which shows the first weight loss of 15 wt $\%$ between 180 and $320{ }^{\circ} \mathrm{C}$, corresponding to the loss of the guest DMF molecules in the pore. The second weight loss starts at around $480{ }^{\circ} \mathrm{C}$, corresponding to the decomposition of ZIF-62
XRPD experiment was performed and the corresponding XRPD patterns are shown in Fig. S1. The positions and intensities of the diffraction peaks only changed slightly during heating (unit cell volume decreased by only $4 \%$ between RT and $350{ }^{\circ} \mathrm{C}$ ) and the structure was retained up to $350{ }^{\circ} \mathrm{C}$. The decomposition of ZIF-62 started at $375{ }^{\circ} \mathrm{C}$ and was completed at $400{ }^{\circ} \mathrm{C}$. A higher decomposition temperature was detected by TGA compared with in situ XRPD, which may be due to the shorter heating time during the TGA that resulted in a delay of the removal of the decomposed linkers. The high thermal stability of ZIF-7 and ZIF-62 is important for potential applications in industrial processes. 


\section{Conclusions}

We have studied how the batch composition and the synthesis temperature affect the formation of zeolitic imidazolate frameworks (ZIFs) in the system with hetero-linkers [Zn:Im:bIm] under solvothermal conditions. Two main phases were identified by X-ray powder diffraction to be ZIF-7 and ZIF-62. The effect of the batch composition on the formation of ZIF-7 and ZIF-62 at $110{ }^{\circ} \mathrm{C}$ was constructed into a phase diagram. Both ZIF-7 and ZIF-62 were produced within a wide composition range applying an excess of the linkers.

Our study shows that the batch composition and synthesis temperature affected the crystal sizes of ZIF-7 and ZIF-62 differently. The size of ZIF-7 crystals changed in a non-linear manner with the $\mathrm{bIm} / \mathrm{Zn}$ ratio, while at a certain $\mathrm{Zn}: \mathrm{L}$ ratio, the crystals of ZIF-62 increased in sizes with the decrease of the $\mathrm{Im} / \mathrm{Zn}$ ratio. The influence of the temperature on the crystal size and morphology was relatively small for ZIF-7 but very significant for ZIF-62. A higher temperature gave slightly smaller crystals of ZIF-7 but larger crystals of ZIF-62. Both ZIF-7 and ZIF-62 have high thermal stability, up to at least $350{ }^{\circ} \mathrm{C}$. Our results show that synthesis parameters, such as the batch composition and temperature, may affect the size and morphology of different compounds in different ways. It is important to screen and optimize synthesis conditions for each compound in order to control the outcome of the final product.

Acknowledgments This project is supported by the Swedish Research Council (VR), the Swedish Governmental Agency for Innovation Systems (VINNOVA), and the Göran-Gustafsson Foundation for Nature Sciences and Medical Research. The electron microscope used in this work was supported by the Knut and Alice Wallenberg Foundation. Dr. Charlotte Bonneau is acknowledged for contributing with important advice about the manuscript, Dr. Junliang Sun for solving the structure of ZIF-62 and Prof. Mikael Kritikos for fruitful discussions.

Open Access This article is distributed under the terms of the Creative Commons Attribution License which permits any use, distribution, and reproduction in any medium, provided the original author(s) and the source are credited.

\section{References}

1. A. Phan, C.J. Doonan, F.J. Uribe-Romo, C.B. Knobler, M. O'Keeffe, O.M. Yaghi, Acc. Chem. Res. 43, 58 (2010)

2. K.S. Park, Z. Ni, A.P. Côté, J.Y. Choi, R. Huang, F.J. UribeRomo, H.K. Chae, M. O'Keeffe, O.M. Yaghi, PNAS 103, 10186 (2006)

3. R. Banerjee, A. Phan, B. Wang, C. Knobler, H. Furukawa, M. O'Keeffe, O.M. Yaghi, Science 319, 939 (2008)

4. R. Banerjee, H. Furukawa, D. Britt, C. Knobler, M. O'Keeffe, O.M. Yaghi, J. Am. Chem. Soc. 131, 3875 (2009)

5. K. Li, D.H. Olson, J. Seidel, T.J. Emge, H. Gong, H. Zeng, J. Li, J. Am. Chem. Soc. 131, 10368 (2009)

6. J.C. Ordonez Ma, K.J. Balkus Jr, J.P. Ferraris, I.H. Musselman, J. Membr. Sci. 361, 28 (2010)

7. N. Chang, Z.-Y. Gu, X.-P. Yan, J. Am. Chem. Soc. 132, 13645 (2010)

8. H. Bux, F. Liang, Y. Li, J. Cravillon, M. Wiebcke, J. Caro, J. Am. Chem. Soc. 131, 16000 (2009)

9. M.C. McCarthy, V. Varela-Guerrero, G.V. Barnett, H.-K. Jeong, Langmuir 26, 14636 (2010)

10. S.R. Venna, M.A. Carreon, J. Am. Chem. Soc. 132, 76 (2010)

11. Y-.Y. Liu, E.-P. Hu, E.A. Khan, Z.-P. Lai, J. Membr. Sci. 353, 36 (2010)

12. Y. Liu, G. Zeng, Y. Pan, Z. Lai, J. Membr. Sci. 379, 4 (2011)

13. H. Bux, A. Feldhoff, J. Cravillon, M. Wiebcke, Y.-S. Li, J. Caro, Chem. Mater. 23, 2262 (2011)

14. B. Wang, A.P. Côté, H. Furukawa, M. O’Keeffe, O.M. Yaghi, Nature 453, 207 (2008)

15. O.M. Yaghi, H. Hayashi, R. Banerjee, PCT Int. Appl. WO 2008140788 A1 (2008)

16. J. van den Bergh, C. Gücüyener, E.A. Pidko, E.J.M. Hensen, J. Gascon, F. Kapteijn, Chem. A Eur. J. 17, 8832 (2011)

17. C. Gücüyener, J. van den Bergh, J. Gascon, F. Kapteijn, J. Am. Chem. Soc. 132, 17704 (2010)

18. Y. Li, F. Liang, H. Bux, W. Yang, J. Caro, J. Membrane Sci. 354, $48(2010)$

19. Y.-S. Li, F.-Y. Liang, H. Bux, A. Feldhoff, W.-S. Yang, J. Caro, Angew. Chem. Int. Ed. 49, 548 (2010)

20. S.R. Venna, J.B. Jasinski, M.A. Carreon, J. Am. Chem. Soc. 132, 18030 (2010)

21. J. Cravillon, R. Nayuk, S. Springer, A. Feldhoff, K. Huber, M. Wiebcke, Chem. Mater. 23, 2130 (2011)

22. G.M. Sheldrick, Acta Crystallogr. A64, 112 (2008) 